\title{
As tecnologias digitais nos percursos de sucesso acadêmico de estudantes não tradicionais do Ensino Superior ${ }^{1}$
}

\author{
Marta Pinto ${ }^{2}$ \\ ORCID: 0000-0001-8271-2818 \\ Carlinda Leite ${ }^{2}$ \\ ORCID: 0000-0001-9960-2519
}

\section{Resumo}

As tecnologias digitais têm sido reconhecidas como mediadoras importantes nos processos de ensino e aprendizagem na Educação Superior. Tendo essa situação por referência, este artigo apresenta uma investigação que estudou o uso das tecnologias digitais, nesse nível de ensino, por estudantes não tradicionais, como suporte à construção de aprendizagens e ao desenvolvimento de competências de autonomia na organização de percursos orientados para o sucesso acadêmico. Foram foco de estudo estudantes dos cursos de História e de Engenharia de Informática e Computação da Universidade do Porto, por se considerar que quem escolhe esses cursos poderá ter competências digitais distintas. Os dados, recolhidos por entrevistas, foram interpretados por análise de conteúdo. Essa análise permitiu identificar dois tipos de tecnologias: as não ofıciais, usadas pelos estudantes por iniciativa própria; e as tecnologias ofıciais, oferecidas pela instituição ou sugeridas pelos docentes. As tecnologias não oficiais são usadas entre estudantes, sobretudo na criação e gestão de espaços virtuais para a comunicação e cooperação na partilha de materiais de estudo em diversos formatos multimédia. Por outro lado, as tecnologias oficiais focam sobretudo o uso para o acesso a materiais de estudo disponibilizados pelo docente e a comunicação com o mesmo. Relativamente à relação do uso das tecnologias com o sucesso acadêmico dos estudantes não tradicionais, o estudo demonstrou que elas são um suporte à autonomia do estudante na gestão e flexibilidade do tempo e locais dedicados ao estudo, à comunicação entre pares e com o docente, e ao acesso ubíquo a materiais de estudo, à sua pesquisa e partilha.

\footnotetext{
1- Este trabalho é apoiado por fundos nacionais, por meio da Fundação para a Ciência e a Tecnologia, IP, do Ministério da Ciência, Tecnologia e Ensino Superior (FCT/MCTES) e pelo Fundo Social Europeu (FSE), por meio do Programa Operacional do Capital Humano (POCH) de Portugal 2020 (bolsa com a ref. SFRH/BPD/116440/2016). É ainda apoiado por fundos nacionais por meio da FCT/MCTES, no âmbito do Programa Estratégico do CIIE (ref. UID/CED/00167/2013).

2- Universidade do Porto, Porto, Portugal. Contatos: mpinto@fpce.up.pt; carlinda@fpce.up.pt.
} 


\section{Palavras-chave}

Tecnologias digitais - Ensino superior - Estudantes não tradicionais - Autonomia Sucesso acadêmico.

\section{Digital technologies in successful academic itineraries of Higher Education non-traditional students}

\section{Abstract}

Digital technologies have been recognised as important mediators in teaching and learning processes in Higher Education. Taking this as a reference, this article presents a research that studied the use of digital Technologies, at this level of learning and by non-traditional students, as a support to the construction of learning and the development of skills for autonomy in the organisation of itineraries oriented towards academic success. Students from History and Computer Engineering courses at University of Porto were the target group of the study, because these courses may have students with distinct digital competences. The data, collected through interviews, were interpreted by content analysis. This analysis allowed the identification of two types of technologies: the unofficial technologies, used by the students on their own initiative; and the official technologies, offered by the institution or suggested by professors. The unofficial technologies are used among students, mainly in the creation and management of virtual spaces for communication and cooperation, for sharing study materials in various multimedia formats. On the other hand, the use of official technologies focuses mainly on the access to study materials made available by the professor and to communicate with the lecturer. Regarding the relationship between the use of technologies and the academic success of non-traditional students, the study has shown that it supports student autonomy, management of the time and place where they study, the communication between peers and with professors, and the ubiquitous access to study materials which they search and share.

\section{Keywords}

Digital technologies - Higher education - Non-traditional students - Autonomy - Academic success.

\section{Introdução}

As tecnologias digitais têm constituído um dos focos de uma nova geração de políticas de educação na União Europeia, sendo um dos eixos prioritários da Agenda 2020. Além disso, têm assumido um papel relevante enquanto suporte aos processos de 
aprendizagem ativos e autônomos dos estudantes do Ensino Superior (ES). Nesse domínio, em linha com o que é esperado pelo Processo de Bolonha, há uma valorização dos processos de aprendizagem ativos e autônomos dos estudantes, baseado no desenvolvimento de competências, nomeadamente as associadas à aprendizagem ao longo da vida (EURYDICE, 2009; MONTEIRO, 2012; VEIGA; MAGALHÃES; AMARAL, 2015; DECLARAÇÃO DE BOLONHA, 1999). De igual modo, é reconhecido que nas últimas décadas chegaram ao ES estudantes com situações e necessidades muito diversas, nomeadamente estudantes não tradicionais (ALMEIDA et al., 2012; AMORIM, 2017), isto é, estudantes que correspondem a uma primeira geração que frequenta esse nível de ensino, frequentemente ingressando no ES após alguns anos de interrupção dos seus estudos. Contudo, os estudantes desses diversos perfis vivem imersos em medias digitais em todos os aspectos da sua vida e continuarão a usá-las nas suas experiências de integração e participação acadâmica e social (BATES, 2016).

Nessa contradição de situações, alguns estudos relativos à situação em Portugal revelam que as tecnologias digitais, quando usadas pelos estudantes como suporte de interação com a instituição onde realizam a formação, são as plataformas de ambientes virtuais de gestão da aprendizagem, de que dispõe a maioria das instituições de ensino superior em Portugal (POMBO et al., 2016; GOMES et al., 2011a, 2011b; DUARTE; GOMES, 2011; LEITE; LIMA; MONTEIRO, 2009). Essas plataformas são as tecnologias digitais mais reconhecidas e dominantes nas instituições de ensino superior, com um forte investimento para a sua implementação (OECD, 2007). Contudo, outras tecnologias como as de comunicação interpessoal também são, muitas vezes, utilizadas pelos estudantes no suporte à aprendizagem (MORAIS, 2012), o que indica possibilidades de um uso de ferramentas que combinam fins pessoais e fins acadêmicos (POMBO et al., 2016; BATISTA, 2011).

Estudos internacionais também identificaram quais as tecnologias digitais que os estudantes do ensino superior usam com mais frequência em contexto de aprendizagem formal e para fins pessoais (ARMSTRONG; FRANKLIN, 2008; CONOLE; ALEVIZOU, 2011; CONOLE, 2014; BULLEN; MORGAN; QAYYUM, 2011; REDECKER; PUNIE, 2010; SANGRÀ, 2008). Infere-se, pois, que o uso de tecnologias tem vindo a ser apropriado em vários contextos de vida das pessoas em geral. No que à educação e formação diz respeito, as tecnologias digitais baseadas na internet, como são, por exemplo, as ferramentas Web 2.0 ou as redes sociais, constituem recursos capazes de promover interações entre estudantes e docentes em contexto de ensino e aprendizagem formal, principalmente porque são ferramentas que podem ser usadas, simultaneamente, pelos alunos nas suas vidas sociais cotidianas e também como suporte aos processos de aprendizagem, proporcionando mais partilha e autonomia pessoal (NORMAN et al. 2013; SLEEMAN; LANG; LEMON, 2016).

A utilização dessas tecnologias pode ter efeitos no tempo dedicado à interação entre estudantes e docentes, continuando para além das paredes da sala de aula, em um processo de envolvimento, partilha de conteúdos e materiais de estudo e de comunicação. Dispositivos como smartphones, tablets, leitores eletrônicos e apps móveis têm-se destacado como ferramentas emergentes de apoio à aprendizagem no ensino superior (ARESTA; PEDR0; SANTOS, 2015; STEVENSON; HEDBERG, 2017; GIORGIEVA; SMRIKAROV; GEORGIEV, 2011), apoiando e transformando as interações entre docentes e estudantes e entre pares (BECKER et al., 2017). Todas essas ferramentas sustentam a criação de ambientes híbridos 
de aprendizagem, fundindo situações de formação presencial com blended learning e mobile learning (ARESTA; PEDRO; SANTOS, 2015).

Investigações com foco no uso de tecnologias digitais por estudantes não tradicionais têm sido reduzidas, contudo, há alguns estudos que referem à valorização que esses estudantes fazem do seu uso como suporte à aprendizagem, alertando para a necessidade de adaptar e selecionar as tecnologias a usar tendo em consideração os níveis de competências digitais. Indicam, ainda, que em estudantes com esse perfil acadêmico esses níveis são frequentemente baixos (KELLY et al., 2009; GACHAGO; LIVINGSTON; IVALA, 2016; PADILLA-CARMONA; SUÁREZ-ORTEGA; SÁNCHEZ-GARCÍA, 2016). Importa, antes de apresentarmos alguns desses estudos, caracterizar a que tipo de estudantes nos estamos a referir.

Em Portugal, por legislação publicada em 2005 (Lei n. 49/2005, de 30/08) foi definido que:

[...] têm acesso ao ensino superior: a) os maiores de 23 anos que, não sendo titulares da habilitação de acesso ao ensino superior, façam prova de capacidade para a sua frequência através da realização de provas especialmente adequadas, realizadas pelos estabelecimentos de ensino superior; b) os titulares de qualificações pós-secundárias apropriadas.

Essa forma de acesso ao ensino superior, regulamentada posteriormente no tipo de provas a prestar, tem vindo a garantir a presença, em cursos do ensino superior, de “estudantes não tradicionais" e que neste texto são designados por "maiores de 23" (23+).

Quanto a estudos que focam estudantes do ensino superior com esse perfil, Dolch; Zawacki-Richter (2018) dão conta de uma investigação acerca do uso de tecnologias entre estudantes tradicionais e não tradicionais do ensino superior na Alemanha, entre 2012 e 2015, que permitiu concluir que ambos os tipos de estudantes dedicam um tempo aproximado ao uso de tecnologias digitais suportadas pela internet, a partir da universidade ou de casa. Referem, no entanto, a existência de uma diferença: os estudantes não tradicionais usam mais frequentemente ferramentas e serviços de suporte ao ensino a distância e menos tecnologias como as redes sociais. Outros estudos focam as percepções e competências para o uso dessas tecnologias por esse grupo de estudantes não tradicionais, em uma investigação realizada entre 2010 e 2012, com estudantes com idades entre os 25 e os 55 anos, maioritariamente trabalhadores estudantes. Concluíram que a maioria acede a tecnologias digitais pela internet em casa ou no trabalho (PADILLA-CARMONA; SUÁREZ-ORTEGA; SÁNCHEZ-GARCÍA, 2016) e que as ferramentas mais utilizadas para apoio ao estudo universitário, por quase metade dos estudantes em ambos os casos, são os ambientes de gestão das aprendizagens e as redes sociais. Concluíram, ainda, que os estudantes revelavam atitudes positivas de percepção do seu uso, com a maioria a preferir realizar cursos onde efetivamente tenham de usar tecnologias digitais, e usando-as sobretudo para aceder a conteúdos de estudo, tratamento e partilha de informação.

Esses estudos, associados à experiência que temos de investigações semelhantes, mostram que as tecnologias são ferramentas incontornáveis de gestão e suporte aos processos de ensino e aprendizagem fazendo parte integral dos processos formais e 
informais de aprendizagem. Nesse processo, tais ferramentas assumem um papel relevante, nomeadamente pelas condições que oferecem aos estudantes para a ampliação do acesso à informação, aquisição e partilha de conhecimentos, promoção da sua autonomização, colaboração, participação e interação com os diversos espaços de aprendizagem em registros presenciais e à distância (POMBO et al., 2016; LEITE; LIMA; MONTEIRO, 2009; ARMSTRONG; FRANKLIN, 2008; LAM et al., 2014). Por outro lado, a flexibilidade de participação e a inclusão nos processos de ensino e aprendizagem que as tecnologias digitais permitem, e criam, poderão beneficiar todos os estudantes, sobretudo aqueles com o perfil de estudantes não tradicionais.

Tendo essas ideias por referência, foi realizado o estudo a que este artigo se reporta cuja intenção é contribuir para a produção de conhecimentos acerca do uso de tecnologias como suporte à construção de aprendizagens, ao desenvolvimento de competências de autonomia e à promoção de percursos de sucesso acadêmico por estudantes não tradicionais. Nessa intenção, secundamos Selwyn (2016) quando afirma a necessidade de aprofundar conhecimento sobre como é que a transformação em educação está a ser feita pelos estudantes com recurso aos media digitais, isto é, saber se os modelos de educação tradicional estão ainda a ser seguidos, ou não, e de que forma as tecnologias estão a ser integradas nesses processos.

Nessa linha de questionamento, este artigo dá conta de uma investigação acerca do uso das tecnologias digitais por estudantes não tradicionais que frequentam, na Universidade do Porto, cursos orientados para diferentes perfis de formação acadêmica. Um desses cursos (História) situa-se na área das Humanidades, enquanto outro (Engenharia Informática) se situa na área Tecnológica. Esta investigação recolheu dados de estudantes com esse perfil, isto é, estudantes que acederam ao ensino superior por meio do contingente Maiores de $23(23+)$ e pretendeu saber que tecnologias são usadas e de que forma, na opinião desses estudantes, o seu uso poderá contribuir para a construção de percursos de sucesso acadêmico.

\section{Metodologia}

A opção seguida no estudo acerca do uso de tecnologias digitais por estudantes não tradicionais, 23+, foi ouvir os próprios estudantes acerca desses usos e dos seus efeitos na construção de percursos de sucesso acadêmico. Os cursos frequentados por esses estudantes foram escolhidos por apontarem para perfis com características muito distintas. Nesse sentido, foram selecionados os cursos de História e de Engenharia de Informática e Computação, ambos da Universidade do Porto, por se pressupor que estudantes que escolhem esses cursos têm competências digitais e de uso de tecnologias bastante distintas, e que por isso é importante compreender os usos e eventuais diferenças.

Em uma primeira fase, foi identificado um total de 18 estudantes desses dois cursos que entraram pelo contingente 23+, ao longo de três anos letivos (2015 a 2018). Depois dessa identificação, todos os estudantes foram contactados no sentido de aceitarem uma entrevista. Responderam ao convite cinco estudantes, três do curso de História e dois do curso de Engenharia de Informática e Computação. 
Uma breve caracterização desses estudantes mostra que: a média de idade é de 36 anos; a dedicação ao estudo a tempo integral acontece para três deles (estando dois totalmente dedicados ao estudo universitário e um a conciliar o emprego com o estudo a tempo integral); a dedicação a tempo parcial ao estudo acontece para dois estudantes que conciliam os estudos com o emprego; o grau de formação acadêmica mais elevada dos estudantes, antes de entrarem no curso de ensino superior, é o ensino secundário (ensino médio) para três estudantes, e graduação para dois outros.

Os estudantes revelaram motivações diferenciadas para o ingresso no ensino superior: o gosto pela área de estudo do curso e interesse em aprofundar o seu conhecimento foram motivos mencionados por dois estudantes; a procura de melhores oportunidades de emprego no futuro foi afirmada por três deles.

As entrevistas foram realizadas presencialmente, entre dezembro de 2017 e fevereiro de 2018, à exceção de uma que se realizou on-line, opção que resultou da falta de disponibilidade para que ela fosse presencial, e apoiaram-se em um guia estruturado de questões. Essas entrevistas foram gravadas e transcritas, depois de um consentimento informado, e interpretadas pela técnica da análise de conteúdo (BARDIN, 2009; KRIPPENDORFF, 2003). Para garantir o anonimato, a cada estudante foi atribuído um código de H1 a H3 para os estudantes do curso de História, e E1 a E2 para os estudantes do curso de Engenharia de Informática e Computação.

\section{Tecnologias digitais usadas no suporte à aprendizagem}

Os dados recolhidos dos estudantes do curso de História e do curso de Engenharia de Informática e Computação que entraram pelo contingente 23+ na Universidade do Porto são apresentados em torno de dois focos de análise: o primeiro relativo às tecnologias digitais usadas pelos estudantes e o segundo ao tipo de uso feito por essas tecnologias digitais no suporte à aprendizagem.

Para efeitos de análise, assumimos a definição de tecnologias digitais referida por Selwyn (2016, p. 124, tradução livre):

Tecnologias digitais podem incluir, mas não estão limitadas a: computadores, tablets, smart phones, Facebook, Moodle, serviços online da Biblioteca, Google, Youtube, escrever ensaios no Microsoft Word, etc. Tecnologia digital também permite às pessoas acederem à internet, não só a partir de casa, mas também a partir de qualquer outro local através do uso de dispositivos móveis.

Quanto ao uso das tecnologias no suporte à aprendizagem, foram consideradas todas as atividades que podem conduzir ou contribuir para a aprendizagem formal e informal por parte dos estudantes. 0 resultado da análise das opiniões dos estudantes $23+$ entrevistados revelou que usam tecnologias digitais no suporte à aprendizagem acadêmica, mas também que utilizam tecnologias escolhidas para fins pessoais. Estas últimas são aqui designadas por não oficiais, enquanto as tecnologias sugeridas pelos docentes ou oferecidas pela universidade são designadas por oficiais. De um total de 13 tecnologias digitais identificadas como usadas no suporte à aprendizagem, oito são não oficiais e cinco são oficiais. 


\section{Tecnologias digitais não oficiais}

Das tecnologias não oficiais, a maioria é suportada pela internet como redes sociais, aplicações de comunicação ou o e-mail, enquanto que as tecnologias não suportadas pela internet são softwares em uso como processadores de texto. A internet é utilizada por todos os estudantes entrevistados para fins acadêmicos e nas diversas atividades do processo de aprendizagem. As tecnologias digitais não oficias utilizadas pelos estudantes no suporte à aprendizagem são: Facebook; Processador de texto Word; Vídeos; Documentos eletrônicos como eBook ou eTextbook; Wikipédia; Whatsapp; Dropbox; Software específico.

Das tecnologias não ofıciais mais utilizadas pelos estudantes, encontra-se a rede social Facebook (4 estudantes referem-na), sobretudo no uso de grupos privados que esta rede social permite criar e gerir. Foram dados dois exemplos desse tipo de grupos. 0 primeiro tipo de grupo privado é o mais referido pelos estudantes e é usado para a partilha de informação, conteúdos e materiais de aprendizagem (como, por exemplo, documentos, links úteis, vídeos on-line), e para a comunicação entre estudantes acerca de questões relacionadas com o curso, os professores ou os estudos. 0 segundo tipo de grupo privado identificado é mais restrito e é usado por estudantes que frequentam o mesmo curso e que vivem na mesma cidade, localizada longe da cidade onde se situa a Universidade do Porto. Esse segundo grupo é sobretudo utilizado como espaço de comunicação entre os seus membros. Acerca desse uso foi referido:

Uma delas [tecnologias não oficiais] é o Facebook, onde estamos todos lá. É a principal. [...] e eu estou a adaptar-me aos novos tempos. Eu já usava o Facebook para partilha, mas de outras coisas. (Participante H1).

0 Facebook eu uso muito. Já temos um grupo de História criado. Criado pelo grupo de alunos de História da FLUP de vários anos, onde se partilha e recebe informação. (Participante H3).

Esses resultados são secundados por estudos internacionais, focados no uso de tecnologias por estudantes tanto com perfis de estudantes tradicionais como de estudantes não tradicionais, confirmando uma elevada utilização de redes sociais (DOLCH; ZAWACKIRICHTER, 2018), sobretudo do Facebook (PADILLA-CARMONA; SUÁREZ-ORTEGA; SÁNCHEZ-GARCÍA, 2016), principalmente para atividades de comunicação e colaboração em que os estudantes podem entre si colocar questões, partilhar informações e ideias e trabalhar em conjunto (HENDERSON et al., 2015; HENDERSON; SELWYN; ASTON, 2015).

Há dois tipos de tecnologias que suportam a comunicação entre estudantes, sendo o Facebook (referido por 4 estudantes) o mais usado, seguido do Whatsapp que, apesar de ter sido referido por apenas um estudante, implicou a comunicação entre um grande grupo de outros estudantes pertencentes à mesma turma, como foi referido: "Usamos o Whatsapp. Tiveram de mo instalar (risos), criamos grupos e é por aqui que falamos (Participante H3).”

Esses resultados de uso das redes sociais onde coexistem a partilha de informação e a comunicação entre pares revela que essas ferramentas têm vindo a ser mais frequentemente mobilizadas pelos estudantes, integrando diversas tipologias de uso, o que significa uma evolução relativa ao que foi apontado no estudo de Morais (2012). Esse 
estudo, desenvolvido entre 2010 e 2011, focando os perfis de estudantes tradicionais e de estudantes não tradicionais, concluiu que os estudantes de ensino superior em Portugal evidenciavam três aspetos: o primeiro relacionado com o uso de ferramentas diferentes para partilhar conteúdo e comunicação entre pares; o segundo que os índices de uso de redes sociais em contextos de suporte à aprendizagem eram pouco frequentes; e o terceiro, que o uso de tecnologias que permitem a comunicação entre pares era mais frequente, recorrendo, contudo, a ferramentas utilizadas exclusivamente para esse fim (como, por exemplo, o MSN e o Skype).

Os vídeos na internet (3 estudantes fazem essa referência) são uma tecnologia frequentemente utilizada pelos estudantes que pesquisam vídeos com conteúdos relacionados com os tópicos abordados nas aulas. Esse uso é justificado por, a partir dos vídeos, acederem a outras formas de explicação dos tópicos, o que lhes permite compreenderem e aprofundarem os conhecimentos. Alguns vídeos são partilhados entre estudantes, utilizando para isso os grupos privados de Facebook. Foi referido:

Por vezes até vejo vídeos sobre matérias do curso. [...] Um colega que vai partilhar no grupo do Facebook um vídeo muito bom que responderá a uma das perguntas de um trabalho que temos que fazer a uma das disciplina. (Participante H1).

Com a mesma intenção de uso dos vídeos, a Wikipédia foi igualmente mencionada como uma ferramenta utilizada para consultar informação quando sentem necessidade de esclarecer dúvidas relativas a conteúdos expostos pelo docente na sala de aula, e que não tenham sido bem compreendidos. 0 seu uso foi mencionado por um estudante: "Costumo ir sempre procurar na internet, seja Wikipédia, ver vídeos no Youtube, é o que costumo fazer (participante E1)."

Os vídeos e a Wikipédia têm sido tecnologias não oficiais apontadas pelos estudantes do ensino superior como muito utilizadas para suporte ao estudo. Henderson et al. (HENDERSON; SELWYN; ASTON, 2015), em uma pesquisa que envolveu recolha de dados junto de duas universidades Australianas, durante o ano de 2014, e em que obtiveram respostas de 1658 estudantes tradicionais e estudantes não tradicionais, concluíram que os três tipos de tecnologias anteriormente mencionadas (Facebook, Vídeos, Wikipédia) eram as mais utilizadas por aproximadamente $90 \%$ dos estudantes.

Uma ferramenta igualmente utilizada para partilha de materiais e recursos das aulas é a Dropbox, sendo um espaço criado e gerido por estudantes do curso de História, que frequentam anos letivos diferentes. Foi dito: “Dropbox também (usamos) para partilha com a nossa turma e para o curso todo, e outros cursos aqui da FLUP [Faculdade de Letras da Universidade do Porto] (participante H1)”. Esta ferramenta, mencionada por um estudante, mostrou o seu uso no apoio ao estudo, permitindo a partilha de pesquisas feitas por outros colegas, ou ainda para constituir documentos como, por exemplo, digitalizar apontamentos das aulas, como mostra o seguinte depoimento: "digitalizamos texto e apontamentos para partilhar uns com os outros” (Participante H1).

Outras ferramentas como o processador de texto Word, mencionado por três estudantes, ou software específico, mencionado por um estudante, foram referidos como usados no suporte à produção de trabalhos sujeitos a avaliação pelos docentes. 


\section{Tecnologias digitais oficiais}

No que refere-se às tecnologias oficiais como suporte ao trabalho acadêmico, ou seja, as tecnologias sugeridas pelos docentes e/ou oferecidas pela universidade, foram identificados cinco tipos usados pelos estudantes no suporte à aprendizagem: e-mail institucional; documentos eletrônicos como eTextbook e PowerPoint; sites de pesquisa bibliográfica; sistema de gestão da aprendizagem Moodle; software específico de uma unidade curricular.

O e-mail institucional foi a tecnologia oficial e institucional referida como utilizada por todos os estudantes. Usam-na com a finalidade de comunicar com os docentes e para receber informação institucional, como foi referido: "0 Webmail é mais para usar com os professores" (Participante E1); "Recebo os mails da comunidade no meu e-mail institucional e são posteriormente e automaticamente reencaminhados para o meu e-mail pessoal. Sintome bastante próximo e informado do que vai acontecendo" (Participante E2).

No estudo desenvolvido por Dolch; Zawacki-Richter (2018), o uso do e-mail institucional foi a tecnologia que mais evidenciou um uso crescente ao longo dos anos (2012-2015), e por estudantes não tradicionais. A familiaridade de uso dessa ferramenta também pode ser apontada como um aspecto facilitador da suas utilização para fins acadêmicos, assim como para situações que exigem mais formalidade (BULLEN; MORGAN; QAYYUM, 2011).

Das tecnologias suportadas pela internet, três estudantes referiram consultar documentos e referências documentais usando para o efeito sites de pesquisa bibliográfica sugeridos pelos docentes. 0 uso dessa ferramenta foi considerado muito útil para o apoio ao estudo ao longo de todo o curso, como mostram as seguintes opiniões:

É muito raro os professores utilizarem tecnologia, exporem imagens. Quando utilizam é sobretudo no $1^{\circ}$ ano para nos mostrarem a biblioteca, mostrar os livros e como se pesquisava....as bases de dados, encontrar artigos. (participante $\mathrm{H} 2$ ).

“[...] os profs fizeram essas referências. Sites fidedignos e que têm um conjunto de teses de investigação a nível mundial" (Participante H1). Também no estudo desenvolvido por Henderson et al. (2015), o uso de repositórios foi percepcionado pelos estudantes, como útil, por responder a requisitos apresentados pelos docentes e por garantir a quantidade e qualidade da informação à qual acedem.

Três estudantes entrevistados revelaram aceder a outros documentos eletrônicos disponibilizados pelos docentes, como, por exemplo, textos ou materiais de apoio às aulas acedendo para isso ao ambiente de gestão da aprendizagem Moodle, frequentemente utilizado como espaço de suporte a algumas unidades curriculares. 0 acesso a essa plataforma permitiu também o desenvolvimento de atividades propostas e dinamizadas pelos docentes, e o acesso a informação de gestão das unidades curriculares, como foi referido: 
Só agora no $3^{\circ}$ ano é que temos um professor que aposta nas tecnologias. Temos no Moodle, nós só estamos a utilizar este ano ..., ele (o professor) põe 10 perguntas, põe materiais tratados como documentos. (Participante H2).

Estudos acerca do ensino superior em Portugal apresentam o Moodle como espaço de acesso a conteúdos educativos, informação e conteúdos relacionado com a avaliação das aprendizagens e que é facultado pelos docentes como suplemento às aulas (MORAIS; BATISTA; RAMOS, 2011; DUARTE; GOMES, 2011).

Outras tecnologias oficiais escolhidas pelos professores e utilizadas pelos estudantes são softwares específicos de apoio a unidades curriculares. Esses softwares foram referidos por um estudante como sendo de uso obrigatório e central em uma unidade curricular, embora tenha sido esse mesmo estudante que referiu também usar software específico não oficial. Referiu: “[...] vamos ter que trabalhar em C++ e Visual Studios. É uma ferramenta obrigatória que vamos ter que usar. Não é ‘vocês podem utilizar isto', mas é 'vocês têm de utilizar isto"”. (Participante E1).

\section{Percepção dos estudantes quanto à contribuição do uso das tecnologias para o sucesso acadêmico}

No prefácio da publicacão Ensino Superior-(in)sucesso académico, Tavares (2000, p. 8) refere que:

Por sucesso académico entendemos não apenas o sucesso escolar ou educativo, mas também o sucesso pessoal, social e comunitário que deverá atingir o estudante universitário durante o tempo da sua vida no interior da academia.

Na mesma publicação, Alarcão (2000, p.19) apresenta como quatro fatores de sucesso acadêmico: o aluno, o currículo, o professor e a instituição. Assumindo esses fatores de base, analisamos nas entrevistas dos estudantes não tradicionais as suas ligações ao uso das tecnologias digitais. Essa análise permitiu identificar que a distribuição desses fatores é a traduzida no Quadro 1.

Quadro 1- Fatores de sucesso acadêmico em relação com o uso de tecnologias digitais

\begin{tabular}{|c|c|c|c|}
\hline Aluno & Currículo & Professor & Instituição \\
\hline $\begin{array}{l}\text { >Autonomia na gestão do estudo } \\
\text { > Cooperação entre estudantes }\end{array}$ & $\begin{array}{c}>\text { Qualidade de recursos } \\
\text { didáticos }\end{array}$ & $\begin{array}{c}>\text { Despistagem de desajustes e } \\
\text { dificuldades do estudante }\end{array}$ & $\begin{array}{c}>\text { Existência de espaços digitais } \\
\text { de estudo }\end{array}$ \\
\hline
\end{tabular}

Fonte: Elaboração das autoras.

Nos fatores de sucesso acadêmico relacionados com o estudante, foi possível identificar a referência à autonomia na gestão do estudo, isto é, uma competência em linha com o que é desejado pela política de educação que justificou o Processo de Bolonha 
iniciado a partir de legislação publicada em Portugal em 2006 (Decreto-Lei n. ${ }^{0}$ 74/2006 de 24 de Março). 0 uso das tecnologias digitais não ofıciais e as oficiais, percecionadas como contributo para a autonomia dos estudantes, prende-se com as possibilidades de uma gestão personalizada do local e tempo dedicado ao estudo. Para o perfil dos estudantes não tradicionais, frequentemente associado a pessoas que conciliam o estudo universitário com o trabalho, a possibilidade de gerir o tempo e o espaço de estudo em casa ou nos intervalos no local de trabalho é tida como um fator importante que pode contribuir para o seu sucesso acadêmico.

Essa inferência mais justifica-se por sabermos que todos os estudantes entrevistados tinham dispositivos móveis como tablet, computador portátil ou telemóvel (smartphone), com ligação à internet, permitindo-lhes um acesso rápido a espaços e materiais de estudo em diversos formatos, onde e quando quiserem. Disseram-nos os estudantes: "Com o tablet posso estudar em qualquer lugar e a qualquer hora. Posso inclusivamente estudar em pequenos intervalos, no trabalho, que vá fazendo ao longo do dia" (Participante E2); "Acho que seria possível ter sucesso sem acesso às tecnologias, mas seria com mais esforço. Teria que fazer mais deslocações. As tecnologias dão-nos um pouco mais de comodismo, dão-nos mais tempo para estudar" (Participante H1).

Os contextos de aprendizagem dentro e fora da sala de aula são cada vez mais configurados pelo uso de vários dispositivos e tecnologias que beneficiam o acesso massificado à internet pelos estudantes nas suas casas e nas instituições de ensino superior que frequentam (BECKER et al., 2017). No nosso estudo, as opiniões expressas pelos estudantes entrevistados demonstraram a existência de uma forte cooperação entre os estudantes que se reflete sobretudo na comunicação entre pares sobre os conteúdos curriculares, e na partilha de materiais de estudo formais resultantes das suas pesquisas individuais. Esses procedimentos, segundo os estudantes afırmaram, permitem uma interação diferenciada com os conteúdos e um aprofundamento posterior aos momentos da aula, como é afirmado no seguinte excerto de um dos entrevistados: "Como vantagem (de uso das tecnologias) o rápido acesso aos conteúdos da cadeira” (Participante E2).

Nesses processos de comunicação, foi possível identificar como um dos fatores de sucesso relacionados com o uso de tecnologias digitais o recurso ao e-mail entre estudante e docente para o apoio ao esclarecimento de dúvidas, tal como demonstra o excerto:

Temos que mandar uma mensagem aos professores quando podemos passar lá para tirar umas dúvidas. E eles se tiverem tempo passamos lá e tiramos umas dúvidas, ou podemos enviar-lhes um e-mail (com as dúvidas) e ver se eles respondem. (Participante H1).

Esta referência está em linha com o que foi concluído em outros estudos (HENDERSON et al., 2015; MORAIS, 2011) acerca do uso das tecnologias oficiais para esse fim. Para os fatores de sucesso relacionados com o currículo, foi identificado um, que se prende com a qualidade dos recursos didáticos (ALARCÃO, 2000), e para o qual contribui, quer o uso de tecnologias digitais oficiais, quer das não oficiais que permitem o acesso alargado a diversos materiais de estudo, incluindo materiais fornecidos pelos docentes como 
e-books, textos diversos e softwares específicos, e materiais selecionados por estudantes e partilhados entre pares.

Nos fatores de sucesso relacionados com a integração institucional dos estudantes, foi possível verificar como benéficos o acesso a ambientes virtuais de gestão da aprendizagem (Moodle) e administrativa, sendo usados para acesso a materiais de estudo, receber e enviar e-mails institucionais, e efetuar ações relacionadas com registros acadêmicos. Essa situação justifica o investimento que as instituições de ensino superior têm feito para garantir a oferta desses ambientes institucionais (OECD, 2007).

As tecnologias usadas para participar na comunidade acadêmica revelaram-se limitadas ao uso do e-mail, na sua funcionalidade exclusiva de receber informação; por exemplo receber informação da faculdade ou associação de estudantes, tal como mostram os seguintes excertos dos entrevistados: "É por e-mail, mais por aí. E depois é pelos corredores, pelos cartazes. No e-mail temos uma carrada de coisas, e às vezes é mais difícil” (Participante H2).

Recebo os mails da comunidade no meu e-mail institucional e são posteriormente e automaticamente reencaminhados para o meu e-mail pessoal. Sinto-me bastante próximo e informado do que vai acontecendo. (Participante E2).

0 e-mail, no estudo que realizamos, continua a ser visto como uma ferramenta oficial e formal de proximidade com a instituição de ensino superior, mas muito utilizada pelos estudantes não tradicionais, tal como concluíram também Dolch; Zawacki-Richter (2018).

A análise desses depoimentos revela que as tecnologias digitais são utilizadas como acesso à informação, quer sobre o processo individual enquanto estudante, quer para receber informação institucional acerca de acontecimentos, eventos, isto é, informação acadêmica, sobretudo enviada para faculdade ou associação de estudantes, mas também como meio que favorece o acesso à informação e à construção de aprendizagens.

\section{Conclusões}

Os estudantes não tradicionais 23+ entrevistados, cujos perfis se previam ser diferentes no que se refere às tecnologias digitais usadas no suporte à aprendizagem, tendo em conta a especificidade dos cursos que frequentam (História e Engenharia de Informática e Computação), revelaram ser similares quanto às competências de autonomia para esse uso e às tecnologias digitais ofıciais e não-oficiais utilizadas. Todos os estudantes revelaram enquadrar-se em um perfil de utilizadores com competências de pesquisa, avaliação, arquivo e partilha de informação em diversos formatos multimédia, e com a capacidade de estabelecer interações diferenciadas de comunicação entre pares.

As tecnologias oficiais são utilizadas privilegiadamente no que é solicitado pelos docentes. 0 amplo uso das plataformas de gestão da aprendizagem, como o Moodle, e cuja oferta tem resultado de um forte investimento institucional (HENDERSON et al., 2015; POMBO et al., 2011; OECD, 2007), serve sobretudo para acesso aos materiais de apoio ao estudo disponibilizado pelos docentes (PADILLA-CARMONA; SUÁREZ-ORTEGA; 
SÁNCHEZ-GARCÍA, 2016). 0 uso do e-mail institucional acontece quando há necessidade de comunicação com os docentes. Os usos dessas tecnologias oficiais acontecem estritamente para fins acadêmicos e são pouco promotores do desenvolvimento de competências de autonomia pelos estudantes.

Para além do uso de tecnologias digitais oficiais, o uso de tecnologias não oficiais mostrou a capacidade de os estudantes selecionarem por iniciativa própria ferramentas que lhes permitem criar e gerir espaços virtuais de cooperação na partilha de materiais de estudo e de comunicação. A menção pelos estudantes ao uso de ferramentas como redes sociais mostra que essas são as mais frequentemente usadas simultaneamente para fins acadêmicos e pessoais, gerindo os estudantes, no seu cotidiano, esses dois espaços que ganham assim uma presença ubíqua.

A rede social Facebook foi a ferramenta não oficial mais referida pelos estudantes, usada para partilha de informação, colaboração e comunicação, confirmando resultados de estudos internacionais que identificaram ser essa uma ferramenta proeminentemente usada por estudantes tradicionais e estudantes não tradicionais (HENDERSON et al., 2015, HENDERSON; SELWYN; ASTON, 2015). O uso de tecnologias não oficiais pode indicar que os estudantes apresentam competências de autonomia na seleção e uso das ferramentas mais adequadas para as suas necessidades de estudo, juntando competências de comunicação e cooperação na partilha de informação e conteúdo.

Do estudo realizado emergiu claramente um perfil de estudante com mobilidade, capaz de recorrer às diversas tecnologias para apoiar o seu estudo, em qualquer momento e lugar, servindo-se para isso do uso de dispositivos móveis, sobretudo o computador portátil pessoal, o que confirma as tecnologias móveis como tendências emergentes no ensino superior (STEVENSON; HEDBERG; 2017; GIORGIEVA; SMRIKAROV; GEORGIEV, 2011). 0 estudante com mobilidade reflete a existência de ambientes híbridos de aprendizagem que ou são ambientes presenciais, ou são ambientes que combinam o presencial e à distância, ou ainda serão ambientes mobile learning (ARESTA; PEDRO; SANTOS, 2015). Esse fator de flexibilidade do espaço de estudo virtual ou presencial, e de gestão do tempo de estudo suportados pelas tecnologias, foi identificado como um fator promotor de sucesso acadêmico, sobretudo relevante na vida de estudantes com o perfil de $23+$ que frequentemente conciliam o estudo, em tempo parcial, com um emprego.

0 estudo permitiu ainda concluir que o uso das tecnologias no suporte à aprendizagem indica ser mais promotor de autonomia quando as ferramentas são escolhidas pelos estudantes, permitindo que esses defınam e criem formas próprias de interação, comunicação e cooperação nos espaços digitais. Foi ainda possível compreender que o uso das tecnologias digitais oficiais no suporte à aprendizagem dentro da sala de aula está frequentemente associado a práticas pedagógicas mais apoiadas na transmissão de informação e no acesso a materiais de estudo. Contudo, nomeadamente porque facilitam o acesso alargado à informação, esse uso também tem efeitos positivos ao nível do sucesso acadêmico e de autonomia através desse acesso.

Face às conclusões a que chegamos, o estudo pode contribuir para a discussão sobre o modo como as tecnologias digitais estão a ser usadas pelos estudantes não tradicionais 
no ensino superior, e de como são incentivados e autores, em nome próprio, do seu uso no suporte à aprendizagem.

Concluímos também que é importante trazer para a reflexão acadêmica a necessidade de investigações que procurem ir além da identificação do uso das tecnologias como suporte da aprendizagem e que, nesse sentido, permitam aprofundar o conhecimento sobre a participação educacional potenciada pelo uso de tecnologias digitais, como é proposto por Selwyn (2016). Em nosso entender, é igualmente necessário e importante considerar as necessidades diferenciadas dos estudantes que chegam ao ensino superior, nomeadamente no que diz respeito aos estudantes não tradicionais, cujo acesso a esse nível de ensino se tornou uma prioridade internacional.

\section{Referências}

ALARCÃO, Isabel. Para uma conceptualização dos fenómenos de insucesso/sucesso escolares no ensino superior. In: TAVARES, José. Ensino superior: (in)sucesso académico. Porto: Porto Editora, 2000. p. 13-23.

ALMEIDA, Leandro et al. Democratização do acesso e do sucesso no ensino superior: uma reflexão a partir das realidades de Portugal e do Brasil. Avaliação, Sorocaba, v. 17, n. 3. p. 899-920, nov. 2012.

AMORIM, José Pedro. Mature students' access to higher education: a critical analysis of the impact of the 23+ policy in Portugal. European Journal of Education, Glasgow, v. 53, n. 3, p. 393-413, 2018.

ARESTA, Mónica; PEDRO, Luís; SANTOS, Carlos. Mobile learning and higher education: a theoretical overview. Journal of Mobile Multimedia, Gistru, v. 11, n. 1-2, p. 147-156, Apr. 2015.

ARMSTRONG, Jill; FRANKLIN, Tom. A review of current and developing international practice in the use of social networking (Web 2.0) in higher education. Franklin Consulting, Alexandria, Virginia, Sept. 2008.

BARDIN, Laurence. Análise de conteúdo. Lisboa: Edições 70, 2009.

BATES, Tony. Teaching in a digital age: guidelines for designing teaching and learning. Vancouver: Tony Bates Associates, 2015.

BATISTA, João. 0 uso das tecnologias da comunicação no ensino superior. 2011. 245 p. Tese (Doutoramento em Informação e Comunicação em Plataformas Digitais) - Universidade de Aveiro, Aveiro, 2011.

BECKER, Samantha Adams et al. NMC horizon report: 2017 higher education edition. Austin: The New Media Consortium, 2017.

BULLEN, Mark; MORGAN, Tannis; QAYYUM, Adnam. Digital learners in higher education: generation is not the issue. Canadian Journal of Knowledge and Technology, Ottawa, v. 37, 1, p. 1-24, 2011.

BOLOGNA DECLARATION. The Bologna declaration of 19 June 1999. Bologna: [s. n.], 1999. 
CONOLE, Gráinne. The use of technology: online distance education: Towards a Research Agenda, Walton Hall, p. 217, 2014.

CONOLE, Gráinne; ALEVIZOU, Panagiota. A literature review of the use of Web 2.0 tools in higher education. York: HEA Academy, 2011.

DOLCH, Carina; ZAWACKI-RICHTER, Olaf. Are students getting used to learning technology? Changing media usage patterns of traditional and non-traditional students in higher education. Research in Learning Technology, Oxfordshire, v. 26, p. 2038, 2018.

DUARTE, Joaquim Alberto Marques; GOMES, Maria João. Práticas com a moodle em Portugal. In: CONFERÊNCIA INTERNACIONAL DE TIC NA EDUCAÇÃO, 7., 2011, Braga, 2011, Braga. Actas da... Braga: Centro de Competência da Universidade do Minho, 2011. p. 871-882.

EURYDICE. Ensino superior na Europa 2009: evolução do Processo de Bolonha. Lisboa: GEPE, 2009.

GACHAGO, Daniela, LIVINGSTON, Candice, IVALA, Eunice. Podcasts: a technology for all? British Journal of Educational Technology, Kingdom, v. 47, n. 5, p. 859-872, 2016.

GEORGIEVA, Evgenya S.; SMRIKAROV, Angel S.; GEORGIEV, Tsvetozar S. Evaluation of mobile learning system. Procedia Computer Science, Durham, v. 3, p. 632-637, 2011.

GOMES, Maria João et al. Distance learning and e-learning in Portugal: A study of the perceptions, concepts and teaching practices at the Institute of Education. In: EDULEARN11. INTERNATIONAL CONFERENCE ON EDUCATION AND NEW LEARNING TECHNOLOGIES, 2011, Barcelona. Proceedings of... Barcelona: [s. n.], 2011a. p. 2617-2623.

GOMES, Maria João et al. Práticas de e-learning no Instituto de Educação da Universidade do Minho: um estudo exploratório. Indagatio Didactica, Aveiro, v. 3, 3, p. 19-43, 2011 b.

HENDERSON, Michael; SELWYN, Neil; ASTON, Rachel. What works and why? Student perceptions of 'useful' digital technology in university teaching and learning. Studies in Higher Education, Routledge, v. 42, n. 8, p. 1567-1579, 2015.

HENDERSON, Michael et al., Rachel. Students' everyday engagement with digital technology in university: exploring patterns of use and 'usefulness'. Journal of Higher Education Policy and Management, Kingdom, v. 37, n. 3, p. 308-319, 2015.

KELLY, Mary et al. A multi-method study to determine the effectiveness of, and student attitudes to, online instructional videos for teaching clinical nursing skills. Nurse Education Today, Stirling, v. 29, n. 3, p. 292-300, 2009.

KRIPPENDORFF, Klaus. Content analysis: an introduction to its methodology. Beverly Hills: Sage, 1980. LAM, Paul et al. Disciplinary difference in students' use of technology, experience in using eLearning strategies and perceptions towards eLearning. Computers \& Education, Oxford, v. 73, p. 111-120, 2014. 
LEITE, Carlinda; LIMA, Lurdes; MONTEIRO, Angélica. 0 trabalho pedagógico no ensino superior: um olhar a partir do Prémio Excelência E-Learning da Universidade do Porto. Educação Sociedade \& Culturas, Porto, n. 28, p. 71-91, 2009.

MONTEIRO, Angélica. 0 Processo de Bolonha e o trabalho pedagógico em plataformas digitais: possíveis implicações. In: MONTEIRO, António et al. (Ed.). Educação online: pedagogia e aprendizagens em plataformas digitais. Santo Tirso: De Facto, 2012. p. 45-54,

MORAIS, Nídia. 0 género e o uso das tecnologias da comunicação no ensino superior público português: um estudo sobre a influência do gênero na perceção dos estudantes sobre o uso de tecnologias da comunicação na aprendizagem. 2012. Tese (Doutoramento em Informação e Comunicação em Plataformas Digitais) Universidade de Aveiro, Aveiro, 2012.

MORAIS, Nídia Salomé; BATISTA, João; RAMOS, Fernando. Caracterização das actividades de aprendizagem promovidas através das tecnologias da comunicação no ensino superior público português. Indagatio Didactica, Aveiro, v. 3, n. 3, p. 6-18, 2011.

NORMAN, Helmi et al. A review on the use and perceived effects of mobile blogs on learning in higher educational settings. Asian Social Science, Ontario, v. 10, n. 1, p. 209-222, 2013.

OECD. Organisation for Economic Co-operation and Development. E-learning in tertiary education: where do we stand. [S. I.]: OECD, 2007. (Policy brief; p.1-8).

PADILLA-CARMONA, Maria Teresa; SUÁREZ-ORTEGA, Magdalena; SÁNCHEZ-GARCÍA, Maria Fe. Digital inclusion of mature students: analysis of their attitudes and ICT competences. Revista Complutense de Educacion, Madrid, v. 27, n. 3, p. 1229-1246, 2016.

POMBO, Lúcia et al. The use of communication technologies in higher education in Portugal: best practices and future trends. In: MARCELINO, Maria José; MENDES, António José; GOMES, Maria Cristina Azevedo. ICT in education: multiple and inclusive perspectives. Cham: Springer International, 2016. p. 1-20.

PORTUGAL. Lei n 49/2005 de 30 de Agosto: lei de bases do sistema educativo. Lisboa: [s. n.], 2005.

REDECKER, Christine; PUNIE, Yves. Learning 2.0 promoting innovation in formal education and training in Europe. In: WOLPERS, Martin et al. (Ed.).; EUROPEAN CONFERENCE FOR TECHNOLOGY-ENHANCED LEARNING: Sustaining TEL: from innovation to learning and practice (EC-TEL), 5., 2010, Barcelona. Proceedings of the... Berlin: Heidelberg: Springer, 2010. p. 308-323.

SANGRÀ, Albert. The integration of information and communication technologies in the university: models, problems and challenges. = La integració de les TICs a la universitat: models, problemes i reptes. 2008. (Unpublished Phd) - Universitat Rovira i Virgili, Tarragona, Spain Tarragona, 2008.

SLEEMAN, Jade; LANG, Catherine; LEMON, Narelle. Social media challenges and affordances for international students: bridges, boundaries, and hybrid spaces. Journal of Studies in International Education, Washington, DC, v. 20, n. 5, p. 391-415, 2016. 
SELWYN, Neil. Is technology good for education? Cambridge: Polity Press, 2016.

STEVENSON, Michael Eric; HEDBERG, John Gordon. Mobilizing learning: a thematic review of apps in K-12 and higher education. Interactive Technology and Smart Education, Bingley, v. 14, n. 2, p. 126-137, 2017.

TAVARES, José. Ensino Superior:(in) sucesso académico. Porto: Porto Editora, 2000.

VEIGA, Amélia; MAGALHÃES, António; AMARAL, Alberto. Differentiated Integration and the Bologna Process. Journal of Contemporary European Research, London, v. 11, n. 1, p. 84-102, 2015.

Recebido em: 23.11.2018

Revisões em: 26.02 .2019

Aprovado em: 09.04.2019

Marta Pinto é investigadora de pós-doutoramento com foco no uso de tecnologias digitais por estudantes do ensino superior no suporte à aprendizagem. Membro integrado do Centro de Investigação e Intervenção Educativas.

Carlinda Leite é professora catedrática e professora emérita da Universidade do Porto, desde 2019, e investigadora sénior do Centro de Investigação e Intervenção Educativas. Desde 2009, é perita da Agência de Avaliação e Acreditação do Ensino Superior (A3ES) na área de formação de professores e de Ciências da Educação/Educação. 